\title{
Entwicklung sprachlicher Fähigkeiten von Kindern und Jugendlichen im Bildungskontext
}

\section{$Z f E$}

\author{
Ingrid Gogolin • Meinert A. Meyer
}

Online publiziert: 19. August 2014

(C) Springer Fachmedien Wiesbaden 2014

Es gehört zu den zahlreichen PISA-Folgen, dass die Bedeutung sprachlicher Fähigkeiten für den Bildungserfolg in die Aufmerksamkeit von Bildungsforschung und interessierter Öffentlichkeit gerückt wurde. Obwohl es eine reiche Tradition der Forschung über sprachliche Entwicklung gibt, sind viele Positionen in diesem Feld hoch umstritten. Insbesondere sind etliche Annahmen und Aussagen über Sprachentwicklung im Bildungskontext nach wie vor eher spekulativ. Sie beruhen auf vielfach durchaus plausiblen theoretischen Extrapolierungen, abgeleitet z. B. aus Analysen der Häufigkeit sprachlicher Phänomene oder der Gradierung sprachlicher Komplexität, kaum jedoch auf empirisch untermauerten Beobachtungen.

Die Sprachentwicklung in der frühen Kindheit ist vergleichsweise gut erforscht, wenngleich auch hier durchaus deutliche Kontroversen bestehen. Zu den umstrittenen Aspekten gehört z. B. die Frage, ob die Aneignung des grammatischen Grundgerüsts in der frühen Kindheit noch in relativer Unabhängigkeit von den Lebensbedingungen geschieht, unter denen ein Kind aufwächst, oder ob auch hier bereits soziale Herkunft und kulturelles Kapital weichenstellenden Einfluss besitzen. Ebenfalls kontrovers diskutiert wird das Thema Zwei- und Mehrsprachigkeit. Einerseits gilt es als gesicherte Erkenntnis, dass in der frühen Kindheit die Aneignung einer zweiten oder weiteren Sprache besonders, leicht' vonstattengeht. So wird etwa in einer jüngst publizierten wissenschaftlichen Stellungnahme zu den biologischen, psychologischen, linguistischen, soziologischen und ökonomischen Bedingungen frühkindlicher

Prof. Dr. Dr. h.c. I. Gogolin (凶) · Prof. Dr. M. A. Meyer

Fakultät für Erziehungswissenschaft, Universität Hamburg,

Von-Melle-Park 8,

20146 Hamburg, Deutschland

E-Mail: gogolin@uni-hamburg.de

Prof. Dr. M. A. Meyer

E-Mail: Meinert.Meyer@uni-hamburg.de 
Sozialisation betont, dass frühe Zweisprachigkeit keine Gefährdung der sprachlichen Entwicklung bedeute; es sei, im Gegenteil, bei Kindern, die einsprachig aufwachsen, ratsam, mit dem Vermitteln einer zweiten Sprache möglichst schon vor dem Schuleintritt zu beginnen (Deutsche Akademie der Naturforscher Leopoldina e. V. 2014, S. 12). Andererseits aber wird das Leben unter Zwei- oder Mehrsprachigkeitsbedingungen vielfach als Risikofaktor gewertet. Diese Einschätzung ist insbesondere in Untersuchungen verbreitet, die sich um Bildungsteilhabe und Bildungserfolg drehen.

Während die Sprachentwicklung in der frühen Kindheit, jenseits aller kontroversen Auffassungen, relativ viel Forschungsaufmerksamkeit erfuhr, gilt dies nicht für die weitere Sprachentwicklung, die sich an der Bildungsbiographie entlang vollzieht, also ab dem Eintritt in eine Bildungsinstitution und über den Verlauf der Bildungskarriere. Insbesondere mangelt es im deutschsprachigen Raum an Untersuchungen, die sich aus der Perspektive der Bildungsforschung um Antworten auf die Frage bemühen, wie sich Sprachentwicklung unter dem Einfluss der gezielten Eingriffe durch die Bildungsinstitutionen vollzieht. Dieses Problem ist in der Vergangenheit in verschiedenen Kontexten bearbeitet worden. So wurde, besonders intensiv in den 1960er und 1970er Jahren, die Frage untersucht, ob und wie die Zugehörigkeit zu einer dialektsprechenden Gruppe das Lernen in der Standardvariante erschwert, die in der Schule verlangt wird. In Deutschland fand dieses Thema seit den 1980er Jahren jedoch kaum mehr Beachtung. Seit den 1970er Jahren wird (auch) in der Erziehungswissenschaft das Problem des Lernens und der Sprachentwicklung unter Zwei- oder Mehrsprachigkeitsbedingungen erforscht. Damit befasste sich aber vorwiegend die Community der auf Folgen der Migration für Bildung und Erziehung spezialisierten Wissenschaftler(innen).

Erst mit dem „PISA-Schock“ wurde die grundlegende Bedeutung, die sprachliche Fähigkeiten für das Lernen überhaupt besitzen, auch in der deutschsprachigen Erziehungswissenschaft und Bildungsforschung breiter wahrgenommen. Es entwickelten sich Forschungsinitiativen, die zur Schließung von Wissenslücken über Zusammenhänge von Sprachentwicklung in ein- und mehrsprachigen Konstellationen beitragen sollen. Aus solchen Initiativen sollten sich die Beiträge zum Thementeil dieses Heftes speisen. Im Entstehungsprozess zeigte sich jedoch, dass das Schwerpunktthema für einige Projekte zu früh kam. Weitere interessante Beiträge zum Thema, die etwa auf der Grundlage von Daten aus dem Nationalen Bildungspanel erarbeitet werden oder auf Forschungsarbeiten beruhen, die im Rahmen von Schwerpunkten der Förderinitiative Bildungsforschung des Bundesministeriums für Bildung und Forschung realisiert werden, werden deshalb nach und nach als freie Beiträge in der ZfE erscheinen.

Im Themenschwerpunkt dieses Heftes sind nun lediglich drei Beiträge versammelt. Der Stichwortartikel von Ingrid Gogolin gibt einen Überblick über den Stand der Forschung zum Thema Sprachentwicklung im Bildungskontext. Nach einer Einbettung in den internationalen Stand der Forschung und Entwicklung konzentriert sich der Beitrag auf Forschungsprojekte der jüngeren Zeit aus dem deutschen Sprachraum, und er schließt mit einem Ausblick auf Fragestellungen, für die in absehbarer Zeit substanzielle Ergebnisse zu erwarten sind. Der Beitrag von Ellen Bialystok und Greg Poarch gibt ebenfalls einen Forschungsüberblick. Hier stehen Untersuchungen im Zentrum, die sich mit der Sprachentwicklung bilingualer Menschen über die Lebenszeit befassen. Nach dem Stand der Forschung ist evident, dass 
Zweisprachigkeit nicht nur in der frühen Sprachentwicklung, sondern auch im Alter kognitive Vorteile mit sich bringt. Der dritte Beitrag von Julia Knoepke, Tobias Richter, Maj-Britt Isberner, Johannes Neumann und Yvonne Neeb befasst sich mit den Voraussetzungen für erfolgreiches Lesenlernen. Auch dieses Problem ist in internationalen Untersuchungen, insbesondere mit englischsprachigen Kindern, breiter erforscht. Die Autoren fragen sich in der vorgestellten Studie, ob und inwieweit die mit englischsprachigen Lernenden gewonnenen Ergebnisse auf deutschsprachige Grundschulkinder übertragbar sind. Diese Frage konnten sie im Rahmen einer querschnittlichen Untersuchung von Zweit- und Viertklässlern untersuchen, deren Ergebnisse im Beitrag vorgestellt werden.

Abgerundet werden die Beiträge zum Themenschwerpunkt durch zwei Sammelrezensionen. Insgesamt bieten die Beiträge des Thementeils einen ersten Einblick in ein erwachendes Feld der Bildungsforschung, aus dem in der näheren Zukunft noch viele interessante grundlegende, aber auch anwendungsrelevante Ergebnisse zu erwarten sind.

\section{Literatur}

Deutsche Akademie der Naturforscher Leopoldina e. V. (Federführung) (Hrsg.) (2014). Frühkindliche Sozialisation. Biologische, psychologische, linguistische, soziologische und ökonomische Perspektiven. Halle (Saale), Nationale Akademie der Wissenschaften Leopoldina. 